\title{
On-line processing of textual illustrations in the visuospatial sketchpad: Evidence from dual-task studies
}

\author{
PETER KRULEY, SONIA C. SCIAMA, and ARTHUR M. GLENBERG \\ University of Wisconsin, Madison, Wisconsin
}

\begin{abstract}
We present evidence that the comprehension of illustrated text makes use of the visuospatial sketchpad component of working memory. The results from three experiments demonstrate that the comprehending of texts that are accompanied by pictures interferes with the performance of a spatial short-term memory task more than does the comprehending of texts that have no pictures. A fourth experiment demonstrates that the interference is found only when there is a requirement to comprehend the text; that is, the simple presentation of the texts and their pictures did not degrade performance on the spatial task. Finally, we show that the comprehension of illustrated texts does not differentially interfere with a verbal short-term memory task. These results are consistent with the claim that illustrations accompanying a text encourage the formation of a spatial mental model with the use of the working memory's sketchpad.
\end{abstract}

Pictures increase comprehension of texts in virtually all situations (Levie \& Lentz, 1982). Our goal was to investigate one component of the process by which this might occur, the use of the visuospatial component of working memory (Baddeley, 1992) to construct spatial mental models. We tested this idea in a series of experiments in which we used concurrent task methodologies. Our basic finding was that the comprehending of texts with pictures disrupted the performance of a spatial concurrent task, but had no effect on the performance of a verbal concurrent task. Not only was this disruption selective in regard to the concurrent task that was disrupted, it also occurred only within the context of a comprehension task.

In most theories of text comprehension, it is agreed that comprehension requires the construction of a referential representation of the meaning of the text: a representation that includes the objects and events being described. Such a representation adds to the literal meaning of the text by incorporating relevant world knowledge. In different theories, referential representations have been given various names, such as situational representations (Perrig \& Kintsch, 1985) and mental models (Johnson-Laird, 1983). A referential representation represents what a text

This research was funded in part by Air Force Office of Scientific Research Grant F49620-92-J-0310 to P.K., and by grants to A.M.G. from the Air Force Office of Scientific Research, 89-0367, and The University of Wisconsin-Madison Graduate School, Project 940109. Preparation of this report occurred while A.M.G. was a research fellow at $\mathrm{ZiF}$, University of Bielefeld, Germany. We thank Nicole Green for many hours spent recording the auditory versions of the descriptions and collecting data. We further acknowledge Michael Masson and an anonymous reviewer for helpful suggestions, including their elaboration of the issue of equal concurrent task difficulty. Requests for reprints may be directed to A. M. Glenberg, Department of Psychology, University of Wisconsin, 1202 West Johnson Street, Madison, WI 53706. is about rather than the text itself (Glenberg, Meyer, \& Lindem, 1987), so that the structure of the objects being described takes precedence over the structure of the text. Our conception of mental models (Glenberg, Kruley, \& Langston, in press) differs from other referential representations in that we take seriously the claim that mental models are analogical. Thus, we are particularly interested in texts that describe spatial layouts or that describe dimensions that are isomorphic to spatial layouts. In these domains, a mental model is hypothesized to consist of representational elements arrayed in the spatial medium of the visuospatial component of working memory.

Support for this formulation is provided by some recent research. Glenberg and Langston (1992) considered several ways in which pictures could aid comprehension, including that pictures simply repeat information, that pictures motivate careful reading, that pictures provide an alternative code for some of the information in the text (dual-code theory, Paivio, 1986), and that pictures facilitate the construction of mental models. The Glenberg and Langston data supported the mental model alternative by demonstrating that, when reading is accompanied by pictures, the structure of the cognitive representations mirrors that of the situations described, rather that the structure of the text. This demonstrates that pictures enabled subjects to appreciate the structure of what was described rather than how it was described. However, this only goes part of the way in supporting our framework; we would like more direct evidence of on-line visuospatial processing.

Glenberg and Kruley (1992) hypothesized that pictures enhance on-line processing during text comprehension. In one of their experiments, subjects read texts alone, read texts accompanied by pictures, or read texts that were followed by pictures. Performance on the comprehension test was best when the pictures accompanied the texts, but pic- 
tures had a statistically significant benefit even when they followed reading of the text ("late picture condition") and thus could not have had a substantial effect on line. Yet although there was no evidence uniquely supporting the hypothesis that pictures aid on-line processing, the hypothesis remains plausible. In the present research, we focused on on-line use of the visuospatial component of working memory (WM).

In Baddeley's formulation of WM there are three components: the central executive and two limited-capacity slave systems-the phonological loop and the visuospatial sketchpad. The phonological loop is a verbal medium; the visuospatial sketchpad is a spatial medium. The slave systems operate independently of each other, temporarily storing and processing information. Although there has been research on the role of the phonological loop in text comprehension (Baddeley \& Hitch, 1974), little has been done on the role of the visuospatial sketchpad. Our goal is to uncover the extent to which picture facilitation in text comprehension is due to on-line processing of the picture and text in the visuospatial sketchpad. To the extent that the picture effect on comprehension requires spatial WM, then comprehension of texts with pictures should disrupt the processing of a concurrent task that requires spatial short-term retention. To test this prediction, we used several versions of concurrent task methodology. The underlying premise is that "having found ways of separately disrupting spatial and verbal processing, one can explore the relative contribution of different subsystems to complex tasks" (Baddeley, 1992, p. 558). Our methodology was modeled after Baddeley and Hitch's research on the use of the phonological loop during comprehension. In their experiments (Baddeley \& Hitch, 1974), subjects performed a comprehension task and a memory task concurrently; while listening to a passage, the subjects were required to remember sequences of zero, three, or six digits. There were two conditions: the preload condition and the control condition. In the preload condition, the digits were presented before each sentence of the passage and the subject was to recall the digits after the sentence was completed. In the control condition, the digits were presented after each sentence and recall was immediate. Comprehension was impaired in the preload condition as opposed to the control condition.

In more recent research, Baddeley (1992) has investigated the role of WM in chess-related tasks. Memory for complex chess positions is disrupted by loading the visuospatial sketchpad or the central executive but not the phonological loop. We aimed to find similar effects of loading the visuospatial sketchpad on comprehension of spatial descriptions. In our experiments, the texts (the same as those used in Glenberg \& Kruley, 1992) contained short scientific descriptions of a single object, a part of an organism, or a mechanical device (e.g., the structure of a leaf). Pictures showed the structural relationships between parts of the object in a level of detail typical of a high-school or undergraduate textbook. As in Baddeley and Hitch's studies, there were two concurrent task con- ditions: a preload condition and a control condition. In the preload condition, a display of dots was presented before and after segments of the text. The subjects were required to determine whether the two displays were the same or different. This required the subjects to retain the first dot display in memory while simultaneously listening to the text. In the control condition, the subjects judged whether a majority of the dots was above a center line. Thus the control condition did not require retention, but it resembled the preload condition by requiring attention to the display and the production of a binary response. For half of the texts, a picture was displayed during text presentation.

In comprehension without a picture, the formation of an analogical representation of the situation described by the text (i.e., the formation of a mental model) is not automatic (Glenberg et al., in press). We hypothesize that the presence of a picture should facilitate the formation of a mental model, and in this case, a spatial concurrent task will compete with comprehension processes for the limited processing capacity of the visuospatial sketchpad. Thus, we would expect the picture to impair performance on the concurrent task.

In addition, the spatial concurrent task might diminish the facilitative effect of the picture in either of two ways. The concurrent task could interfere with the formation of a mental model or it could interfere with the long-term storage of the picture in visual memory, either way, impairing performance on a comprehension test. Regardless, if the picture and the text together interfere with the concurrent task more than the unillustrated text does, then there is evidence of on-line spatial processing during comprehension of illustrated text.

\section{EXPERIMENT 1}

Subjects in the preload task condition were required to verify whether a test configuration of dots arrayed within a grid matched a configuration presented earlier, a task likely to be performed with the use of the WM sketchpad. In the control condition, the subjects saw a configuration of dots in a grid and judged whether a majority of the dots was above the grid's center line. Thus the control condition required the production of a binary response, but not the retention of the initial display. Independently of this task manipulation, picture availability was manipulated over two levels: no picture, and a picture that was available during the entire presentation of the description (full-picture condition).

It seemed likely that the type of concurrent task required would interact with picture presence. Specifically, we assumed that the facilitating effect of the picture depends on its being maintained in the sketchpad. In contrast, subjects are assumed to use the sketchpad less intensively in the nopicture than in the full-picture condition; this assumption is consistent with, for example, Glenberg and Langston (1992), who found that when reading in an unfamiliar domain, subjects typically formed mental models only when 
presented with an explicit diagram. Thus, we hypothesized that when a picture was present, it would occupy the sketchpad for a longer time than would any mental models formed in the no-picture condition, so subjects would be forced to shuttle the picture and the dot display repeatedly in and out of the sketchpad, leading to one of three outcomes: (1) poorer performance on the preload version of the concurrent task in the full-picture condition compared to the no-picture condition; (2) a picture presence $x$ concurrent task difficulty interaction in the comprehension data, with decreased picture facilitation in the preload condition; or (3) a combination of (1) and (2).

\section{Method}

Subjects. Residents of the Madison area participated as subjects in exchange for a cash payment. A total of 36 subjects contributed data; 1 subject was replaced when a computer failure invalidated her data.

Materials and Design. The texts used in Glenberg and Kruley (1992) were recorded as digitized speech and stored on a Macintosh II computer. All texts conformed to a single structure of eight

Table 1

Sample Text Used in the Study

\begin{tabular}{cl} 
Segment & \multicolumn{1}{c}{ Text } \\
\hline 1 & $\begin{array}{l}\text { Volcanoes originate deep in the earth. Between } \\
\text { the volcano's origin and its top is a complex } \\
\text { structure of layers. }\end{array}$
\end{tabular}

2 The fumarole is an opening on the surface of the earth.

3 The magma layer is formed by pockets near the center of the earth.

4 The laccolith is found between two main layers of sedimentary rock.

5 The layer between two sedimentary layers is made of igneous rock.

6 As it moves, it causes the ground surface to rise.

7 The opening on the surface of the earth emits gases.

8 Increased pressure within the earth forces these gases to the surface, and the gases can be used an an indicator of an eruption.

\section{Questions}

(From Segments What is true of the laccolith? a. it is 4 and 5): very hot $b$. it is made of igneous rock $c$. it is gaseous $d$. it signals an eruption (Answer: b)

(From Segments What does the fumerole do? a. emits 2 and 7$):$ gases $b$. causes the ground surface to rise $c$. forms pockets $d$. settles between the two main layers of rock (Answer: a)

(Additional Where do volcanoes originate? a. at the question): earth's surface $b$. in oceans $c$. near mountain ranges $d$. deep in the Earth (Answer: b.)

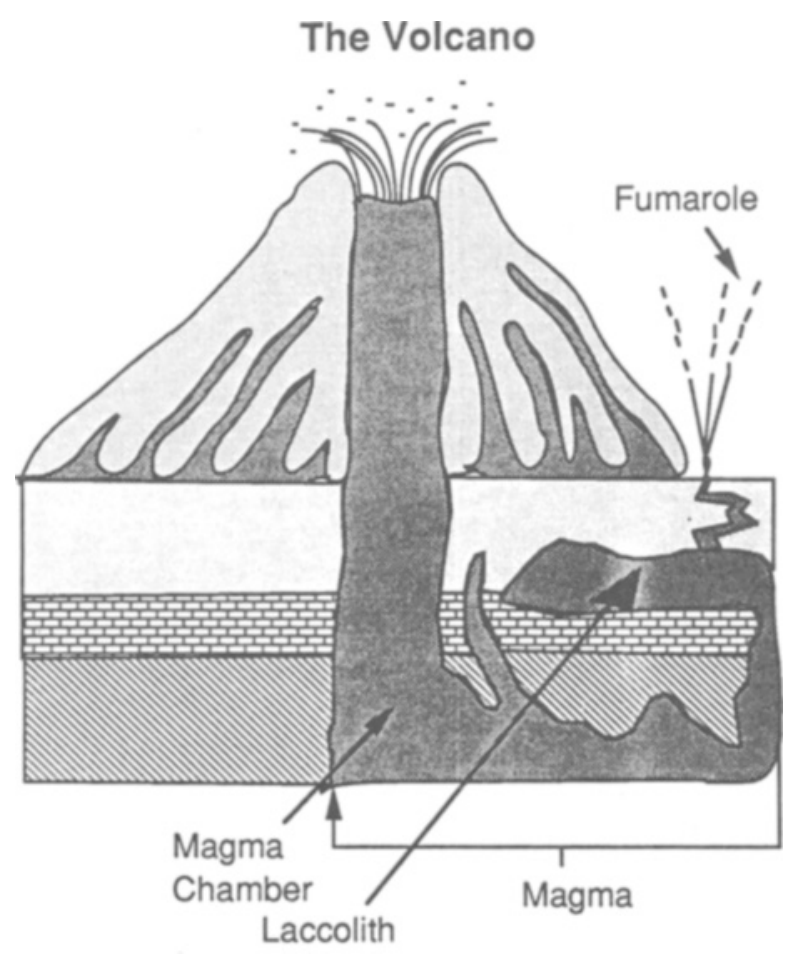

Figure 1. The picture illustrating the description shown in Table 1.

segments, each of which consisted of one or two sentences. The structure (see Table 1) was designed for the study of anaphor resolution; its only relevance to the current series of experiments is that each text contained eight segments. When the texts were recorded, each segment was stored as a separate file on the computer, allowing playback of any single segment independently of the others. A total of 24 scored and 4 practice texts were presented during the experiment. Each text was followed by three multiple choice questions, each with four possible responses, that tested the subject's comprehension of the material. Two of these questions tested the subject's ability to integrate information from different segments of the text, as described in Glenberg and Kruley (1992); in the present experiment, the questions provided a global measure of comprehension. The questions were presented in a random order. A picture was available to illustrate each text; as shown in Figure 1, the pictures displayed the structural relationships between the parts of the object described in the description.

Two levels of picture (none, and a fully available picture) were combined factorially with two levels of the concurrent task (control and preload) in a within-subjects design.

Texts were presented in four blocks, reflecting the four combinations of picture level and dot task difficulty used in the experiment. Each half of the experimental portion of a session consisted of trials with the same level of the dot task; one-half of the texts in each half were presented with no picture, and one-half were presented with a picture. Thus, one possible order of presentation was as follows: (first quarter) control task with picture, (second quarter) control task with no picture, (third quarter) preload task with picture, and (fourth quarter) preload task without picture. The order of presentation was counterbalanced across subjects, with the stipulation that the order of picture conditions was the same in each half of the experiment; thus, for example, the order that would result from reversing the third and fourth quarters above was not used. The counterbalancing system used in the experiment was designed so that each text would be presented equally often in each of the 
four conditions, in the long run. Because the scheme had a repetition cycle of 8 subjects, however, the counterbalancing was not perfect: in the actual experiment, some texts were presented as few as 8 times in a condition, or as many as 10 times.

The display used in the dot task consisted of a rectangular grid, 4 cells by 4 cells, drawn inside a window that nearly filled the computer screen. For test displays, and for initial displays in the preload condition, three round filled (solid black) dots were drawn, each contained within a cell of the grid. For the initial display in the control condition, a grid was drawn without any dots ("empty grid").

The choice of the cells to be filled with dots in the initial displays of the preload condition was made randomly, with the restriction that the three dots could not be colinear. In the preload condition, the choice to present a same or a different trial was also made randomly; each type of trial was given a $50 \%$ chance of occurring. For the dot displays in the different trials, one dot from the initial display was chosen at random and relocated in a randomly selected empty cell adjacent to its original location.

Procedure. The following sequence of events was repeated eight times during the presentation of each text: first, an initial dot display was shown for $667 \mathrm{msec}$; then the display disappeared and subjects heard one segment of the description; then a test display was presented and shown on the screen until the subject responded. In the control condition, the subjects merely had to indicate whether the majority of dots in the test display were above the center line of the grid; if they were, a "yes" response was to be made using the slash (/) key, if not, a "no" response was to be made using the $\mathrm{Z}$ key. The control condition thus did not require subjects to remember the initial display; in fact, an empty grid was used as the initial display in this condition. In the preload condition, responses indicated whether or not the test display was identical to the initial display; the slash key was used to signal a "yes" response (same), and the Z key to signal a "no" response (different). Consequently, the preload condition encouraged the subjects to maintain the initial grid in memory while comprehending one segment of the auditory description. In the no-picture condition, the initial dot display was followed by a blank window during the playing of the text segment; in the full-picture condition, the picture that illustrated the description was displayed during this time. After all eight segments of the text were presented, the picture disappeared-if it had been present-and the three multiple choice questions appeared on the screen one after another. After these questions were answered, a screen display indicated the number of correct answers that had been achieved. The subject was then informed how many dot responses had been correct; if the total was six or fewer out of eight possible, an additional message asked the subject to try harder.

Before the presentation of any scored texts, two practice texts were presented that included the version of the dot task to be performed in the first half of the experiment. One of the practice texts was presented with a picture, and one without. At the start of the second and fourth quarters, a screen message informed the subject of the picture condition that was about to begin. At the start of the second half, a message instructed the subject to summon the experimenter, who gave instructions for the version of the dot task to be used for the remainder of the trials. Two practice texts that incorporated the new version of the dot task were presented at this time.

\section{Results}

Comprehension. Figure 2 shows the mean percent correct on the multiple choice questions in each condition; comprehension was facilitated by picture presence and impaired in the preload dot task condition, but the factors of picture presence and task type did not interact. Analyses of variance were performed with subjects as the random factor (statistics reported as $F_{1}$ ), and using texts as
Performance on the comprehension task

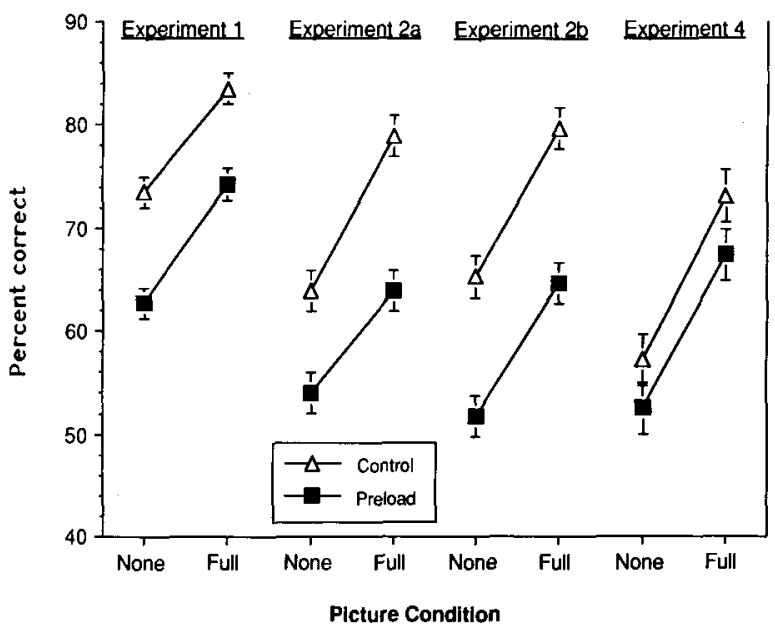

Figure 2. Mean percent correct on comprehension questions in Experiments 1, 2A-2B, and 4 as a function of the task condition and picture presence. Vertical bars extend one standard error above and below the mean, using the mean square for the subject $x$ picture $x$ task interaction as an estimate of within-cell variance.

the random factor $\left(F_{2}\right)$. On the comprehension measure, both main effects were significant ${ }^{1}$ [for task, $F_{1}(1,35)=$ $22.37, M S_{\mathrm{e}}=161.94$ and $F_{2}(1,23)=42.86, M S_{\mathrm{e}}=$ 52.75; for picture, $F_{1}(1,35)=37.54, M S_{\mathrm{e}}=111.90$; and $\left.F_{2}(1,23)=18.00, M S_{\mathrm{e}}=156.91\right]$; the interaction was not $\left[F_{1}(1,35)=0.27, M S_{\mathrm{e}}=80.52\right.$ and $F_{2}(1,23)=$ $\left.0.24, M S_{\mathrm{e}}=85.14\right]$. Thus, examination of the comprehension data alone would not suggest that the pictures and dot displays competed for the sketchpad.

Dot task. Mean percent correct on the dot task is shown in Figure 3; as expected, performance on the control dot task was near $100 \%$ in both picture conditions. Consequently, the variances of the dependent variable were markedly heterogeneous, with variance in the preload dot conditions exceeding that in the control conditions by a factor of approximately 40 . Because such an extreme degree of heterogeneity invalidates the use of analysis of variance (ANOVA) (Maxwell \& Delaney, 1990), and because the modal value in the control conditions coincided with the maximum value, implying a non-normal distribution, statistical significance was assessed using the Wilcoxon signed-ranks test (Siegel \& Castellan, 1988). Analyses were performed on subjects' mean scores (statistics reported as $z_{1}$ ) and on the mean score of each text $\left(z_{2}\right)$. Evaluation of three orthogonal comparisons showed that (1) performance on the preload dot task was significantly better in the no-picture than the full-picture condition $\left(z_{1}=4.51, z_{2}=3.49\right)$; (2) on the control dot task, performance did not differ significantly as a function of picture condition $\left(z_{1}=1.36, z_{2}=1.42\right)$; and (3) overall, performance was higher on the control task than on the preload task $\left(z_{1}=5.24, z_{2}=4.29\right)$. In the dot task data, then, picture presence and task type did interact, consis- 
Performance on the concurrent task

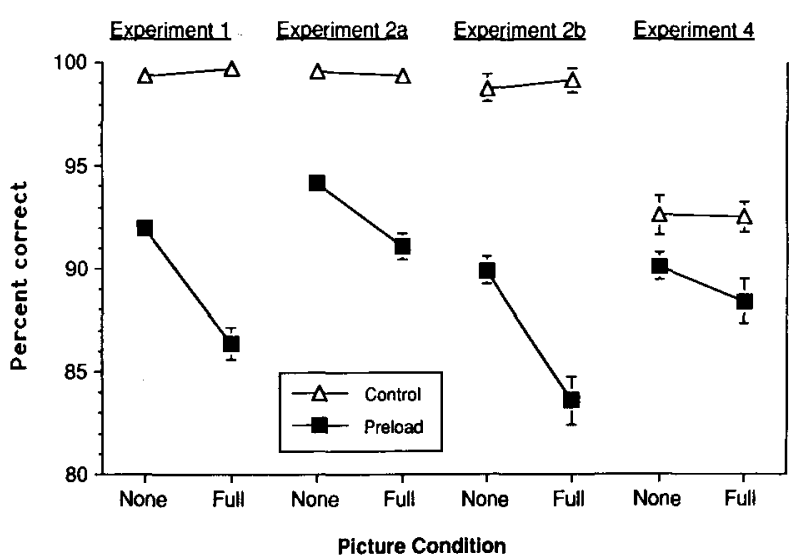

Figure 3. Mean percent correct on the concurrent task in Experiments 1, 2A-2B, and 4. In Experiment 4, the concurrent task required the recall of a string of digits; in the other experiments, it required the memory of a dot display. Because of heterogeneity of variance across conditions, standard errors were computed separately for each cell, on the basis of the within-cell standard deviation of residualized scores (i.e., scores after the removal of betweensubject variance; see Loftus \& Loftus, 1988, p. 427). Where no error bar is shown, the standard error was $<0.43$.

tent with the hypothesis of competition for the WM sketchpad.

\section{Discussion}

Experiment 1 demonstrated that the presence of a picture did interact with the concurrent task, a task that required the use of the visuospatial sketchpad for memory. Although the absence of a significant interaction in the comprehension data indicates that the picture improved comprehension equally regardless of the concurrent task, the dot task data indicate that performance of the preload task was impaired by the presence of a picture during comprehension. Thus, picture presence and concurrent task type can interact; it is reasonable to assume that this interaction depends on the presence of competing memory demands-specifically, the need to use the visuospatial sketchpad. Alternative explanations will be considered in the discussion of Experiments 3 and 4; Experiment 3 tested the hypothesis that the picture interfered perceptually with dot task performance, and Experiment 4 tested whether the effect was specific to situations involving competition for the WM sketchpad.

\section{EXPERIMENTS 2A AND 2B}

Experiment 1 produced evidence that the processes used in performing the dot task and those used during comprehension with a picture compete for the use of the visuospatial sketchpad. In Experiment 1, the effect of this competition was seen in performance on the dot task, with subjects performing less accurately when the picture was present. If comprehension and maintaining the dot display in WM are competing for the sketchpad, then giving the dot task greater emphasis might produce an effect on the comprehension data. Specifically, if subjects attended to the dot task sufficiently to equalize performance across picture conditions, then the competition between the two tasks should appear as a picture $\times$ task interaction in the comprehension data, with pictures having a greater facilitating effect in the control dot task condition. Experiments $2 \mathrm{~A}$ and $2 \mathrm{~B}$ were designed to test whether a shift in motivation would induce subjects to resolve competition for the sketchpad in favor of the dot display, leading to an interaction in the comprehension data. In both experiments, instructions explicitly directed the subjects to give priority to the dot task; Experiment $2 \mathrm{~B}$ differed from Experiment $2 \mathrm{~A}$ in that five dots were used in the dot displays, making the task more difficult and presumably leading to greater competition for the sketchpad.

\section{Method}

Subjects. Subjects in both experiments were introductory psychology students who participated voluntarily in exchange for course credit. In Experiment 2A, 1 subject was replaced for failing to call the experimenter to get the second half of the instructions, and 1 subject's total of 24 correct on the comprehension questions was more than two standard deviations below the overall mean; this subject was omitted from the analyses. A total of 37 subjects provided data for analysis. In Experiment 2B, 27 subjects contributed usable data; 1 subject was replaced for failing to exceed chance performance on the comprehension questions.

Materials and Design. The stimuli consisted of the same 24 experimental and 4 practice texts used in the earlier experiments. The 2 (no picture vs. full picture) $\times 2$ (control vs. preload task) withinsubjects design of Experiment 1 was repeated in both Experiments $2 A$ and $2 B$. In Experiment 2A, the dot displays used were the same in Experiment 1, whereas in Experiment $2 \mathrm{~B}$ all displays other than the empty grid consisted of five filled dots, one to a cell.

Procedure. The experimental procedure repeated that of Experiment 1 , with two critical modifications that were designed to motivate subjects to focus on the dot task. First, the instructions were changed to describe the dot task as the "primary task" of interest; comprehension was described as secondary. To emphasize this order of priority, the instructions included the following paragraph:

You'll probably find that you can't devote your full attention to both the dot display and the recorded text all of the time. When the procedure becomes difficult, make sure to do as well as you can on the dot task, even if it means missing out on some of the text information. That is, the main task that we are interested in is the dot task, so please do your best on it.

The second modification to the procedure involved the manner of providing feedback. Feedback on performance on the dot task was provided in a colorful screen display: a sequence of eight colored dots was revealed, one dot at a time, to indicate accuracy on the eight dot tasks performed during the presentation of a text. Red dots were used to signal incorrect responses and green dots to signal correct ones. In addition, musical tones were played as the dots were revealed. The dot signaling the first correct response was accompanied by the musical tone $\mathrm{C}$, and each subsequent correct response was accompanied by a note that was one step higher in the $\mathrm{C}$ scale. Thus, producing eight correct responses on a text resulted in the playing of a complete $\mathrm{C}$ major scale. When this occurred, the achievement was emphasized by the playing of a $\mathrm{C}$ major triad after the last note. In contrast, the red dot signaling each incorrect 
response was accompanied by a burst of noise. As in the earlier experiments, a screen display informed subjects of the number of correct responses made on the comprehension questions.

As before, the counterbalancing system was designed to assign texts equally often to conditions. In Experiment 2A, counterbalancing was complicated by three events: the total number of subjects was not a multiple of eight, 1 subject's data was discarded after the completion of data collection, and the experimenter assigned an incorrect subject number on one occasion, causing the computer program automatically to assign texts to conditions according to the subject number specified. As a result of these occurrences, some texts were presented as few as 7 times in an experimental condition, or as many as 11 times.

\section{Results}

Comprehension. For both experiments, Figure 2 shows the mean percent correct in each condition. In Experiment $2 \mathrm{~A}$, the main effects of picture and task were again significant [for task, $F_{1}(1,36)=43.68, M S_{\mathrm{e}}=133.16$ and $F_{2}(1,23)=40.62, M S_{\mathrm{e}}=82.86$; for picture, $F_{1}(1,36)$ $=52.06, M S_{\mathrm{e}}=111.72$ and $F_{2}(1,23)=30.38, M S_{\mathrm{e}}=$ 132.59]. The task $\times$ picture interaction only attained significance when texts were used as the random effect $\left[F_{1}(1,36)=1.76, M S_{\mathrm{e}}=145.30\right.$ and $F_{2}(1,23)=6.39$, $\left.M S_{\mathrm{e}}=50.34\right]$. The direction of the interaction was as hypothesized, with a greater picture effect (full picture exceeding no picture) occurring in the control task condition.

In Experiment 2B, both main effects were significant [for task, $F_{1}(1,26)=32.79, M S_{\mathrm{e}}=168.391$ and $F_{2}(1,23)$ $=38.38, M S_{\mathrm{e}}=122.14$; for picture, $F_{1}(1,26)=28.21$, $M S_{\mathrm{e}}=179.21$ and $\left.F_{2}(1,23)=18.96, M S_{\mathrm{e}}=253.69\right]$; the interaction was not $\left[F_{1}(1,26)=0.13, M S_{\mathrm{e}}=108.38\right.$; $\left.F_{2}(1,23)=0.24, M S_{\mathrm{e}}=104.49\right]$.

Dot task. Mean percent correct on the dot task for both experiments is shown in Figure 3. As before, examination of the variances and distributional shapes of the data indicated that the assumptions for an ANOVA were not met, and planned comparisons were performed with the Wilcoxon signed-ranks test. In both Experiment $2 \mathrm{~A}$ and Experiment 2B, the pattern of results was the same as in Experiment 1: performance on the preload dot task was significantly better in the no-picture than in the full-picture condition (Experiment $2 \mathrm{~A}, z_{1}=3.36, z_{2}=2.96$; Experiment $2 \mathrm{~B}, z_{1}=3.54, z_{2}=3.46$ ); on the control dot task, performance did not differ significantly as a function of picture condition (Experiment $2 \mathrm{~A}, z_{1}=0.76, z_{2}=0.94$; Experiment $2 \mathrm{~B}, z_{1}=0.91, z_{2}=1.24$ ), and overall, performance was higher on the control task than on the preload task (Experiment $2 \mathrm{~A}, z_{1}=5.24, z_{2}=4.29$; Experiment $2 \mathrm{~B}, z_{1}=4.46, z_{2}=4.29$ ).

\section{Discussion}

Experiment 2A followed the procedure of Experiment 1 exactly with the addition of explicit motivational changes encouraging subjects to focus on the dot task. Evidently, the motivation instruction was effective: performance on the comprehension questions declined from that obtained in Experiment 1, and performance on the preload dot task improved. The question addressed by the motivation instruction was whether subjects could reserve the use of the visuospatial sketchpad for the dot memory task, applying the sketchpad as an aid in comprehension only when it was not needed for the concurrent task. If subjects had made this priority shift fully, performance on the preload dot task would have been equal in both picture conditions. The results of Experiment $2 \mathrm{~A}$ suggest that subjects partially succeeded in making the shift: the difference between picture conditions in the preload dot task was smaller than in Experiment 1, but still significant. Furthermore, mean performance on the comprehension questions conformed to the pattern of the predicted picture $\times$ task interaction. This interaction was not significant in the analysis by subjects, but was significant in the analysis by texts. When the specifics of the assignment of texts to conditions are considered, it appears that the analysis by subjects is more valid; in the analysis by texts, the means for text-condition combinations were computed over unequal numbers of subjects. Accordingly, the shift in statistical significance from the analysis by subjects to that by texts reflects not only differences in error terms (subjects $\times$ picture $\times$ task vs. texts $\times$ picture $\times$ task), but also a shift in the actual cell means. Fortuitously, the shift was in the direction of a larger interaction in the analysis by texts. On the whole, therefore, Experiment $2 \mathrm{~A}$ provided suggestive evidence of a partial shift in the priority of use of the sketchpad; results on both the dot and comprehension measures shifted in the predicted direction, but the changes failed to affect the statistical significance of the results.

In Experiment 2B, the pattern of results on both measures returned to that obtained in Experiment 1. We included a more difficult version of the dot task in this experiment, with the expectation of increasing the competition for the sketchpad and thereby increasing the magnitude of the effects produced by the change in task priority. Evidently, however, this change had a different effect: faced with a more difficult dot task, subjects abandoned the effort to give it priority at all times. We speculate that our undergraduate student subjects found it difficult at best to ignore the demands of the comprehension questions; under the greater stress of the harder dot task, they may have resorted to devoting attention to the type of work for which they were most accustomed to receiving evaluation. Note, however, that the results of Experiments $2 \mathrm{~A}$ and $2 \mathrm{~B}$ were consistent with the hypothesis that the dot task competes with the use of the picture in comprehension for the visuospatial sketchpad: once again, pictures interfered significantly with performance on the preload dot task.

\section{EXPERIMENT 3}

As we mentioned earlier, the observed result that performance on the dot task decreased when a picture accompanied the description need not be attributed to on-line comprehension processes. Specifically, the presentation of a picture might interfere with the perceptual processes involved in performing the dot task by disrupting the sub- 
ject's visual mental image of the dot display. Alternatively, the mere mention in the description of labeled parts of the picture might instate it automatically into the subject's visuospatial sketchpad, without any comprehension taking place. In Experiment 3, we tested these possibilities by removing the requirement that subjects attempt to comprehend the description: as before, the subjects performed the dot task while hearing auditory descriptions, some of which were accompanied by pictures. This experiment differed from the previous experiments, however, in that no comprehension questions followed the description. The subjects were informed of this fact in advance. An additional change was made to the dot displays; because subjects were likely to perform very accurately on the dot task in the absence of a comprehension requirement, the displays were made more difficult in order to avoid ceiling effects in subject performance. The difficulty level of the task was adjusted individually for each subject, as described below.

Our hypothesis was that pictures would interfere with the concurrent task only when they were required for comprehension. Thus, in this experiment, in which comprehension was not required, pictures should not interfere with the concurrent task. An alternative account for this null finding would be that with no comprehension requirement, subjects might close their eyes or look away from the screen in order to avoid being distracted by the irrelevant picture. Given the need to look at the screen to respond to the test display, and given that subjects could not easily anticipate when this display would be presented, we doubted that many would adopt this strategy. Nevertheless, to rule out this possibility experimentally, we asked the subjects to return to the laboratory for a second session, at which time we administered a picture recognition test.

\section{Method}

Subjects. Forty-two undergraduates participated voluntarily in exchange for course credit. An additional 2 subjects were replaced-
1 because of an error in labeling the keys to be used in responding, and 1 because of a computer failure.

Materials and Design. The stimuli consisted of 20 of the 24 experimental texts used in the earlier experiments; 4 texts on which comprehension scores were either consistently very high or very low were dropped. Dot displays were presented on a rectangular grid of nine cells by nine cells. Three, four, or five of the cells were filled with dots, depending on the difficulty level of the dot task (see below). The single factor manipulated in this experiment was picture presence, with two levels: no picture, and a fully available picture.

Procedure. The experiment consisted of three phases: initial practice on the dot task with feedback, further practice with delayed feedback, and the scored portion of the experiment. Initial practice consisted of four trials of the dot task-subjects saw an initial dot display, heard one segment of a text, then responded to a test display. Each response was immediately identified as correct or incorrect. The dot displays used in this experiment differed from the earlier version of the task in that the pattern of dots might be displaced (translated) from the initial to the test display. The subjects were instructed to treat a pattern that was spatially displaced but that preserved the configuration of the dots (see Figure 4) as a same trial. In the second practice phase, the subjects performed eight dot trials during the presentation of a text with a picture, received feedback on the set of eight trials as a whole, and then performed eight trials during the presentation of a text without a picture. During the scored portion of the experiment 20 texts were presented, 10 with and 10 without pictures (i.e., 80 trials of the dot task were performed in each picture condition). The assignment of texts to conditions was counterbalanced across subjects. Texts were presented in a random order, with the stipulation that every set of two texts, beginning with the first text presented, consisted of one text with picture and one text without, though not necessarily in that order.

We anticipated that subjects would find the dot task very easy in the absence of a comprehension requirement; accordingly, the difficulty of the dot task was adjusted to induce an overall level of accuracy that would permit a valid test of the experimental hypothesis. The dot task could be presented at one of eight levels of difficulty; as shown in Table 2, these varied according to the number of dots in the pattern, the distance that the pattern was displaced from the initial to the test display, and the amount of spread in the pattern. The latter factor refers to the extent of the portion of the grid in which dots were placed; in Figure 4, for example, the spread is five cells by five cells. After every even-numbered text presentation, the difficulty of the dot task was adjusted in order to achieve
(A)

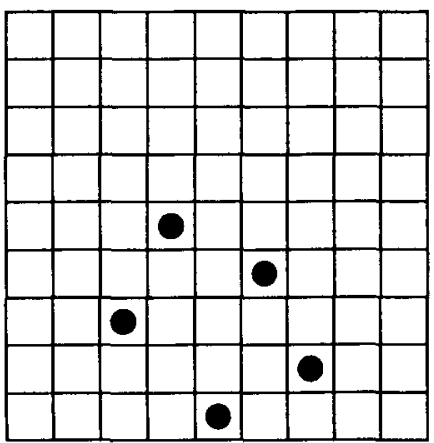

(B)

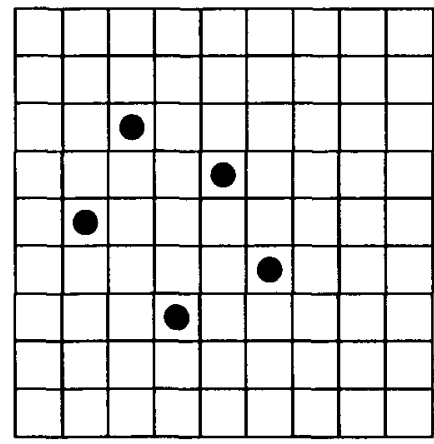

(C)

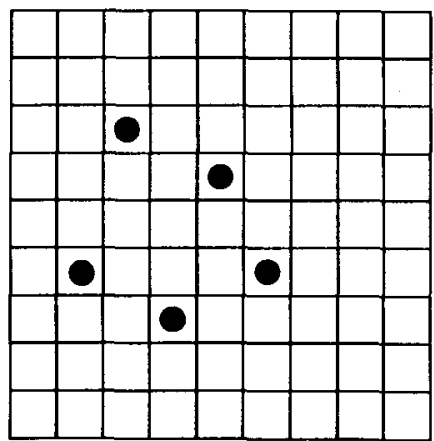

Figure 4. Examples of dot displays used in Experiment 3. If the display shown in panel A were presented initially, the display in panel B would call for a "yes" response (relative positions of the dots are the same as in panel A), whereas the display in panel $\mathbf{C}$ would call for a "no" response (relative positions are different from those in panel $\mathbf{A}$ ). These examples correspond to Difficulty Level 6. 
a target performance of $75 \%$ correct. Accordingly, if a subject had made 12 correct responses out of the 16 possible during the presentation of two texts, the difficulty level was left unchanged. If 11 responses were correct, difficulty was decreased by one level, whereas if 10 or fewer responses were correct, difficulty was decreased by two levels. Similarly, if more than 12 responses were correct, difficulty was increased by either one or two levels. Because each set of two successive texts contained one text in the picture and one in the no-picture condition, the difficulty adjustment affected both conditions equally. The difficulty of the dot task was set to Level 5 for the two practice presentations, then adjusted as described above before the first scored presentation.

Recognition task. After the conclusion of the principal experimental session, the subjects were contacted and asked to return for a second session; those who did return were paid $\$ 1$ for participating. For the recognition test, the 20 pictures that accompanied the texts were displayed one by one, and the subjects were instructed to indicate whether or not they had seen the picture during the original experimental session. Because each subject had heard 10 texts in the picture condition and 10 texts in the no-picture condition, 10 of the pictures had been displayed during the original session and 10 had not.

\section{Results}

Dot task. Performance in the no-picture condition $(M=80.3 \%$ correct $)$ and the full-picture condition $(M=$ $80.4 \%)$ did not differ significantly $\left[F_{1}(1,41)=0.00\right.$, $\left.M S_{\mathrm{e}}=16.73\right]$. The mean difficulty level for all trials was 7.2; Table 2 shows the mean accuracy and the number of trials performed at each difficulty level. With the exception of the highest level (Level 8), accuracy decreased monotonically with increasing level of difficulty, as we intended. Although accuracy was higher at Level 8 than at Level 7, we do not believe that this indicates any problem with the difficulty adjustment. Rather, because most subjects reached Level 8 early in the course of the experiment and performed most of their trials at this level, we believe that the improved performance reflects the effect of extended practice.

Recognition task. A total of 23 subjects returned for the recognition session. Scores on the recognition task ranged from 12 to 19 correct out of 20 possible, with a mean of 15.3. This value differed significantly from the value of 10 correct that would be achieved by chance responding [one-sample $t(22)=13.03, M S_{\mathrm{e}}=0.41$ ]. In an analysis of this task as a signal detection task, mean $d^{\prime}$ was $1.67(s=0.71)$. Although there was no requirement to process the pictures in any way during the initial experimental session, recognition performance was high,

Table 2

Difficulty Levels of the Dot Task, Experiment 3

\begin{tabular}{cccccc}
\hline Level & $\begin{array}{c}\text { Number } \\
\text { of Dots }\end{array}$ & $\begin{array}{c}\text { Distance } \\
\text { to Move }\end{array}$ & Spread & $\begin{array}{c}\text { Number } \\
\text { of Trials }\end{array}$ & $\begin{array}{r}\text { Percent } \\
\text { Correct }\end{array}$ \\
\hline 1 & 3 & 0 & $3 \times 3$ & 0 & \\
2 & 3 & 1 & $3 \times 3$ & 0 & \\
3 & 4 & 2 & $3 \times 3$ & 16 & 100.00 \\
4 & 4 & 2 & $4 \times 4$ & 80 & 88.75 \\
5 & 5 & 2 & $4 \times 4$ & 624 & 88.62 \\
6 & 5 & 2 & $5 \times 5$ & 880 & 80.68 \\
7 & 5 & 2 & $6 \times 6$ & 1648 & 75.97 \\
8 & 5 & 3 & $6 \times 6$ & 3472 & 80.58 \\
\hline
\end{tabular}

ruling out the possibility that subjects had closed their eyes or otherwise ignored the picture. The median delay separating the original experimental session and the recognition session was 50 days. Length of delay correlated negatively with performance on the recognition task $(r=$ $-.43, p<.05$ ), confirming that the task tapped the subjects' memory for the experimental session.

\section{Discussion}

Performance on the dot task did not differ significantly as a function of the presence or absence of a picture. Because our conclusions depend on the acceptance of this null result, it is incumbent on us to present a power analysis of the experiment. Qualitatively, this experiment, which was subjected to an ANOVA and had an $N$ of 42 , had greater power than did the earlier experiments in which nonparametric statistical analyses were performed on smaller samples. Quantitatively, the power advantage can be demonstrated as follows: the smallest picture effect found earlier occurred in Experiment 2A; on the preload version of the dot task, accuracy in the no-picture condition exceeded accuracy in the picture condition by $3.1 \%$. If the mean square error from the ANOVA is used as an estimate of the variance of the mean difference, the power to detect an effect as small as $3.1 \%$ is greater than 99. A difference of $1.8 \%$ could have been detected with a probability of .80 . Consequently, we have great confidence that the picture effect in this experiment was either absent or much smaller than in any of the earlier experiments. In other words, in the absence of a requirement to comprehend, subjects performed equally well on the dot task, regardless of whether the picture was present. Data from the recognition task confirm that subjects looked at the screen during the experiment. In summary, the decrement in performance of the dot task found in the earlier experiments cannot be attributed to the mere display of a picture. Rather, performance on the dot task suffered only when subjects used the picture to comprehend the description.

\section{EXPERIMENT 4}

Experiments 1-3 provided evidence in support of the hypothesis that the presence of a picture during text comprehension encourages processing in the visuospatial component of working memory. In Experiment 1, the presence of a picture interfered with the performance of a spatial concurrent task. To strongly support the hypothesis, we needed to show that the presence of a picture during text comprehension would not interfere with a nonspatial concurrent task. In other words, we needed to show that the interference is specific to the spatial domain.

In Experiment 4, subjects were required to perform a concurrent digit task during text comprehension. In the digit task, four digits were presented (the initial presentation); at a later time two of these digits were re-presented (the test presentation), and the subjects' task was to determine whether the order of the digits in the test presentation was the same as the order of the digits in the initial 
presentation. For example, if the initial presentation consisted of the digits 4259 and the test presentation consisted of the digits 49 , the correct response would be "yes," because 4 occurs before 9 in both the initial presentation and the test presentation, whereas if the test presentation consisted of the digits 92 , the correct response would be "no," because 9 occurs after 2 in the initial presentation and before 2 in the test presentation.

In the preload condition, each segment of the text was embedded in the interval between the initial presentation and the test presentation of the digit task, so subjects had to maintain the digits in memory while listening to the text. As a control condition against which to measure the interference of the preload condition, the initial and test presentations both occurred between segments. Text segments in the control condition were presented before each occurrence of the digit task; there was only a short unfilled interval between the initial presentation and the test presentation.

\section{Method}

Subjects. Introductory psychology students served as subjects in exchange for class credit. A total of 32 subjects contributed data.

Materials and Design. The same texts and comprehension questions were used as in Experiments 1 and 2A-2B.

The digits zero through nine were recorded on the Macintosh computer by a male speaker. Each digit lasted approximately $360 \mathrm{msec}$.

Two levels of picture presence (none, and a fully available picture) were combined factorially with two levels of the concurrent task (control and preload) in a within-subjects design.

A counterbalancing system was designed to present each text equally often in each of the four conditions; each text appeared eight times in each condition.

Procedure. For each segment of the text, the concurrent task was performed once. In the preload condition, four digits were presented (the initial presentation) before each segment of the text; $500 \mathrm{msec}$ after the end of the segment, two of these digits were re-presented (the test presentation). All presentations were auditory. As well as listen to the text, subjects were to determine whether the digits in the test presentation were presented in the same order in both the initial and the test presentations. The response was to be made by using the slash (/) key for "yes," the same order, and the $Z$ key for "no," a different order, on the computer keyboard. The control condition was the same as the preload condition, except that the digit task was performed between segments: The initial presentation occurred $500 \mathrm{msec}$ after each segment of the text; $333 \mathrm{msec}$ later, the test presentation occurred.

As in Experiments 1 and 2A-2B, three multiple choice questions appeared on the screen after the presentation of the description. These questions were followed by screen displays indicating the number of correct answers and the number of correct digit responses.

As in Experiment 1, texts were presented in four blocks reflecting the four combinations of picture level and digit task.

\section{Results}

Comprehension. Comprehension performance is shown in Figure 2. The presence of a picture aided performance on the comprehension task, and the preload version of the digit task caused a decrement in comprehension performance. Both main effects were significant [for picture, $F_{1}(1,31)=47.67, M S_{\mathrm{e}}=160.28$, and $F_{2}(1,23)=27.40$, $M S_{\mathrm{e}}=207.78$; for task, $F_{1}(1,31)=6.79, M S_{\mathrm{e}}=127.78$ and $F_{2}(1,23)=4.87, M S_{\mathrm{e}}=133.33$ ]. There was no sig- nificant interaction between the two factors $\left[F_{1}(1,31)=\right.$ $\left.0.04, M S_{\mathrm{e}}=200.34 ; F_{2}(1,23)=0.05, M S_{\mathrm{e}}=123.33\right]$.

Digit task. Performance on the digit task is shown in Figure 3. There was a main effect of digit task; the preload version of the digit task was harder than the control digit task $\left[F_{1}(1,31)=6.68, M S_{\mathrm{e}}=51.74\right.$ and $F_{2}(1,23)=$ 13.34, $\left.M S_{\mathrm{e}}=19.37\right]$. There was no effect of picture presence $\left[F_{1}(1,31)=1.03, M S_{\mathrm{e}}=24.02\right.$ and $F_{2}(1,23)=$ $\left.0.88, M S_{\mathrm{e}}=21.09\right]$. There was no significant interaction between the two factors $\left[F_{1}(1,31)=1.05, M S_{\mathrm{e}}=20.21\right.$ and $\left.F_{2}(1,23)=1.46, M S_{\mathrm{e}}=10.78\right]$.

Two planned comparisons showed that there was no effect of picture presence on either the preload or the control concurrent task [preload, $F_{1}(1,31)=1.77, M S_{\mathrm{e}}=$ $25.96, F_{2}(1,23)=1.77, M S_{\mathrm{e}}=19.22 ;$ control, $F_{1}(1,31)$ $\left.=0.00, M S_{\mathrm{e}}=18.27, F_{2}(1,23)=0.00, M S_{\mathrm{e}}=12.50\right]$.

\section{Discussion}

The presence of a picture had no effect on performance of the concurrent digit task. This suggests that the interference found in Experiments 1 and 2A-2B was specific to visuospatial processing. As for the conclusions reached from Experiment 3, our contention relies on acceptance of a null hypothesis, so a power analysis is necessary. Again we will demonstrate that this experiment had the power to detect the smallest picture effect found previously-3.1\% in Experiment $2 \mathrm{~A}$. If the mean square error from the ANOVA for the simple effect of picture presence on the preload task $\left(M S_{\mathrm{c}}=25.96\right)$ is used as an estimate of the variance of the mean difference, the power to detect an effect of size $3.1 \%$ is .93 . A difference of $2.5 \%$ could have been detected with a probability of .80 . Although the data in Figure 3 hint at an interaction between the factors of picture presence and task type, little statistical evidence supports such an interaction, and that is particularly impressive given the power to detect small effects. ${ }^{2}$ Hence we can confidently conclude that, although the presence of a picture had a detrimental effect on dot task performance, it had no appreciable effect on digit task performance.

In Experiments 1, 2A, and 2B, performance on the preload version of the concurrent task was impaired in the picture condition relative to the no-picture condition, whereas in Experiment 4, performance on the preload concurrent task did not differ across picture conditions; consequently, we conclude that picture presence interferes with a concurrent task only when that task is visual or spatial in nature. To support this conclusion, it is necessary to show that the nonspatial digit task that we used was at least as difficult as the visuospatial dot task of the earlier experiments. That is, the processing of the illustrated text might fail to interfere with an easy concurrent task simply because the combined resource demands of the two tasks fell within the subject's capacity.

Regarding the question of task difficulty, examination of Figures 2 and 3 indicates that the preload version of the digit task elicited levels of performance comparable to (or worse than) those of Experiments 1, 2A, and 2B on both dependent measures; thus, the two types of con- 
current task appear equally demanding. This pattern of results does not conclusively indicate that the tasks produced equal resource demands, however, given that the experiments were conducted separately. That is, the resource demand needed to induce a given level of accuracy on a concurrent task may differ in different groups of subjects. An alternative index of task difficulty, in which a comparison is made between observations performed by the same subjects, is the difference between comprehension scores in the control and preload conditions. Because the decrement in comprehension in the preload relative to the control condition was smaller in Experiment 4 than in the other experiments (see Figure 2), this comparison favors the interpretation that Experiment 4's digit task was less demanding on resources than the dot task of the other experiments. ${ }^{3}$ In our view, however, the likeliest explanation for this effect is that the control digit task used in this experiment was more demanding than the control dot task used earlier (Experiment 4 was actually performed first of the experiments reported here, so it was not possible to make the control tasks exactly parallel). The difference in the tasks is that the control dot task required only a trivial judgment (whether a majority of dots lay above the center line of a grid), whereas the control digit task required the retention of a sequence of digits. Even though this digit sequence was retained for only a short period of time, and though the task was not concurrent with the comprehension of the text segments, this control task proved more difficult than the control dot task. Thus, although it is possible, on the basis of the comparison discussed above, that the dot task suffered interference from the picture simply because it was more demanding than the digit task, we believe that the crucial point is the apparently equivalent difficulty of the preload versions of the two types of concurrent task. In light of all of these considerations, we conclude that picture presence was found to interfere with a preload concurrent task only when that task was spatial.

\section{GENERAL DISCUSSION}

We addressed two questions in this investigation. First, is the picture processed on line, at the time of comprehension? Second, does the presence of a picture during comprehension of a description affect the use of the visuospatial sketchpad component of WM? In three experiments, subjects were required to remember spatial configurations of dots while comprehending textual descriptions, some of which were presented with and some without an accompanying picture. The availability of a picture during comprehension consistently impaired memory performance on the concurrent task. In Experiment 3, however, subjects were not required to comprehend the descriptions, and the presence of a picture had no effect on dot memory performance. Thus, we conclude that using a picture as an aid in comprehension interferes with performance on the dot memory task; consequently, at least some processing of the picture is being performed on line. As discussed earlier, Glenberg and Kruley (1992) found that recall was significantly higher in the late-picture condition of their Experiment 3 than in the no-picture condition. That is, readers benefited from the availability of a picture even when it was presented after the text had been read and was no longer available for further reading. Although this result is consistent with effects that occur at retrieval rather than during comprehension, two other results require a further mechanism: the superiority of recall in the full-picture as opposed to the late-picture condition (Glenberg \& Kruley, 1992, Experiment 3), and the finding in the present investigation that picture presence has an on-line effect on performance on a spatial concurrent task. We believe that these outcomes reflect the use of the visuospatial sketchpad during comprehension.

In Experiment 4, subjects performed a digit memory task concurrently with the comprehension task. The results resembled those of Experiments 1 and $2 \mathrm{~A}-2 \mathrm{~B}$, in that comprehension was improved by the presence of a picture and impaired by the requirement to maintain a string of digits in memory during listening to the textual description. The results of Experiment 4 differed from the earlier experiments, however, in one crucial respect: there was no statistically significant effect of picture presence on performance of either the preload or the control version of the digit task. Thus, the results obtained across all experiments are strongly consistent with Baddeley's (1986) model of WM, which predicts that pictures and dot displays will be processed in the sketchpad subsystem, but that digit sequences will be processed in the articulatory loop.

One of our expectations was not met: that the effect of competition for the sketchpad could be made symmetricthat it could occur either in the concurrent task data (in the form of diminished performance on the preload task in the picture condition) or in the comprehension data (in the form of decreased picture facilitation in the preload condition). In fact, the interference occurred only in one direction: comprehension using pictures interfered with a spatial concurrent task. Before providing a complete account of the experimental results, we wish to note that this asymmetric interference is consistent with the claim that the tasks compete for the sketchpad. As Norman and Bobrow (1975) reasoned:

Interference can only be observed when a process is operating within its resource-limited region. Note, therefore, that the effects of interference need not be symmetrical. If task $A$ interferes with task $B$, but not the reverse, then it would be incorrect to conclude that one of these tasks does not require processing capacity from the same central pool as the other. On the contrary, interference in either direction implies that both tasks draw resources from the same common pool. (p. 50)

The full pattern of results obtained in the present experiments was as follows: comprehension was impaired during performance of the preload concurrent task, implying that the concurrent task and the process of comprehension competed for processing resources, regardless 
of picture presence; also, picture presence impaired performance of a spatial concurrent task, implying competition for the sketchpad. This pattern of results can be explained on the basis of four factors: (1) excess demands on the sketchpad during comprehension with a picture in the preload dot condition, (2) indivisibility of the comprehension process with respect to utilization of the information in the picture, (3) spare spatial processing capacity during performance of the control concurrent task, and (4) a reduction of the availability or capacity of WM control processes (supervisory attentional system, or SAS) in any divided attention situation. In outline, in the fullpicture preload dot condition, to resolve excess demands on the sketchpad (Factor 1), subjects may either utilize the picture or ignore it, with no middle ground (Factor 2). Evidently, comprehension involves integrating information from the picture with other background knowledge and with information from long-term memory. To follow our motivational instructions by using the information in the picture only when doing so would not interfere with the dot task, subjects would have to exercise the SAS to such a degree that the entire enterprise would be lost. Thus, our purpose might have been achieved by instructing subjects to close their eyes or look away from the screen when things got sticky, but not by instructing them to subdivide WM or attentional capacity. To complete the account, the modest drop in performance of the dot task in the picture condition implies that the comprehension task alone does not exhaust spatial capacity (Factor 3); subjects evidently resolve any conflict for the sketchpad in favor of the comprehension task, but the capacity available after processing the picture is still great enough to yield a high level of accuracy on the dot task. Finally, because comprehension is impaired during the preload task in comparison with the control concurrent task, whether that task is spatial or not, we conclude that the division of attention between two demanding tasks requires active control of the part on the SAS, thereby decreasing its availability for any single task (Factor 4).

The function of the executive process referred to above, the SAS, is to resolve conflicts between concurrent processes. Baddeley (1986) draws on an earlier model of Shallice and Norman to describe how potential conflicts are avoided when schemata are operating simultaneously. Although each schema may be highly automated, it may still be necessary to assign one process a higher priority at any given moment. When possible, this assignment is performed automatically on the basis of predetermined priorities and environmental cues, but when such automatic scheduling would not be accurate or effective, the SAS exercises active control of the scheduling of processes. In Baddeley's model, the visuospatial sketchpad and the articulatory loop constitute subsystems that are driven by the SAS and operate semiautonomously once activated. The model is motivated by findings of differential interference within and across classes of tasks: spatial tasks, for example, interfere with other spatial tasks (in the sketchpad) but not with verbal rehearsal (performed in the articulatory loop). The task used experimentally to stress the SAS is one in which the subject must recite letters or numbers in a random sequence; the production of truly random responses requires a high degree of active control. On the basis of these relationships between WM subsystems, we derived the account of the present results given earlier: the division of attention between the preload concurrent task and the process of comprehension results in general performance decrement because of demands on the SAS, whereas the combination of a spatial concurrent task and an illustrated text specifically stresses the sketchpad.

What properties of the sketchpad suit it for use in text comprehension? In our view, the sketchpad is a generalpurpose spatial representational medium that is used to support mental models. Before considering the role of mental models in comprehension, we will review some evidence supporting the claim that the sketchpad is a general spatial medium-that it represents spatial relationships, rather than serves a specific perceptual or motor function. Baddeley (1986, pp. 112-113) reported an experiment in which performing a spatial task involving audition and motor control interfered selectively with performance on a concurrent task that involved visualization. The former task involved tracking a moving pendulum that emitted sounds: blindfolded subjects were instructed to keep a flashlight beam pointed at the pendulum as it moved. The latter task required the recall of a sequence of steps through a $4 \times 4$ matrix and could be performed by visualizing the number 1 in the first square visited, the number 2 in the second, and so on. Subjects made significantly more errors on the matrix memory task while simultaneously performing the pendulum tracking task than when performing the memory task alone; in contrast, performance on a control memory task that involved no visualization was unaffected by the requirement to track the pendulum concurrently. Thus, a spatial task selectively interfered with performance on another spatial task, although there was little or no overlap in the perceptual and motor demands of the two tasks. Baddeley also reported that a visual, nonspatial task (making brightness judgments) affected the control memory task more than the spatial task, confirming that the sketchpad is not an intrinsically visual medium. Similarly, Taylor and Tversky (1992) found that subjects recalled spatial layouts quickly and accurately regardless of whether they had read survey (i.e., a bird's eye view) or route (i.e., a mental tour from one person's perspective) descriptions. Although their experiment did not specifically implicate WM or the sketchpad, their conclusion that readers form objectcentered representations of layouts described in texts is consistent with the conception of the sketchpad as a general-purpose spatial representational medium.

The robust value of pictures in the comprehension of written texts may be explainable in large part by the capacity of the sketchpad to support mental models that represent a wide variety of situations. Although the sketchpad may have evolved to aid in finding one's way in 
natural environments (see, e.g., Taylor \& Tversky, 1992), it is evident that human comprehenders and reasoners are capable of using its spatial properties in many applications, both spatial and nonspatial. To summarize our position, mental models are situational representations that are temporarily represented and operated on in the WM sketchpad. The models are analogical, in the sense that representational elements are arrayed in spatial configurations, and that the spatial relationships between elements are meaningful for the processes that operate on the model (Glenberg et al., in press). For example, Mani and Johnson-Laird (1982) tested subjects' recall of descriptions of spatial configurations of objects. They found that the level of recall was sensitive to particular kinds of indeterminacies in the descriptions: although linguistically clear, descriptions which might have referred to more than one distinct arrangement of the objects led to decreased recall performance. Thus, Mani and Johnson-Laird concluded that the descriptions were being represented as spatial layouts, rather than as propositions describing relations between objects. The value of pictures and diagrams is that they too are analogical spatial representations. Thus a picture can suggest an appropriate mental model for a text, and perceiving the picture may well serve to instantiate the model in the sketchpad.

The sketchpad also aids in comprehension of nonspatial descriptions, because the use of the sketchpad as a medium for instantiating graphs and figures makes possible the representation of nonspatial information in a spatial form. Glenberg and Langston (1992) tested the memory of subjects for descriptions of four-step procedures, which were presented either with or without a supporting diagram that illustrated the temporal order of the steps. Questions posed after reading the description probed for recall of the temporal relations; the diagram was not available to the subject while the subject was answering the question. When a diagram was available during comprehension, subjects' responses reflected the temporal structure of the procedure being described, consistent with the use of a mental model. In contrast, responses in the no-diagram condition reflected the order in which the steps of the procedure had been described in the text. In the domain of reasoning, Johnson-Laird, Byrne, and Tabossi (1989) found evidence that subjects employed mental models to solve problems of multiple quantification (e.g., All artists are beekeepers; some beekeepers are chemists, etc.). For these problems, subjects evidently formed concrete representations of the situations evoked by the descriptions in the mental model. Thus, comprehenders of texts are able to represent both spatial and nonspatial situations in the sketchpad; given the virtually limitless number of situations that can be represented analogically, the capacity for forming these representations may well explain the consistent value of pictures during comprehension.
BADDELEY, A. (1992). Is working memory working? The Fifteenth Bartlett Lecture. Quanterly Joumal of Experimental Psychology, 44A, 1-31. BADDELEY, A. D., \& HiTCH, G. J. (1974). Working memory. In G. Bower (Ed.). Recent advances in leaming and motivation (Vol. 8. pp. 47-90). New York: Academic Press.

GLENBerG, A. M., Kruley, P. (1992). Pictures and anaphora: Evidence for independent processes. Memory \& Cognition, 20, 461-471

Glenberg, A. M., Kruley, P., \& Langston, W. E. (in press). Analogical processes in comprehension: Simulation of a mental model. In M. A. Gernsbacher (Ed.), Handbook of psycholinguistics. Orlando, FL: Academic Press.

Glenberg, A. M. . \& Langston, W. E. (1992). Comprehension of illustrated text: Pictures help to build mental models. Joumal of Memory \& Language, 31, 129-151.

GlenberG, A. M., Meyer, M., \& Lindem, K. (1987). Mental models contribute to foregrounding during text comprehension. Journal of Memory \& Language, 26, 69-83.

Johnson-Laird, P. N. (1983). Mental models. Cambridge, MA: Harvard University Press.

Johnson-Laird, P. N., BYrne, R. M. S., \& TABossi, P. (1989). Reasoning by model: The case of multiple quantification. Psychological Review, 96, 658-673.

LEVIE, W. H., \& LENTZ, R. (1982). Effects of text illustrations: A review of research. Educational Communication \& Technology Journal, 30 , 195-232.

LofTUs, G. R., \& LofTus, E. F. (1988). Essence of statistics (2nd ed.). New York: Random House.

Mani, K., \& Johnson-LaIRD, P. N. (1982). The mental representation of spatial descriptions. Memory \& Cognition, 10, 181-187.

MaXWELL, S. E., \& Delaney, H. D. (1990). Designing experiments and analyzing data/A model comparison perspective. Belmont, CA: Wadsworth.

Norman, D. A., \& Bobrow, D. G. (1975). On data-limited and resource-limited processes. Cognitive Psychology, 7, 44-64.

PaIvio, A (1986). Mental representation: A dual coding approach. New York: Oxford University Press.

Perrig, W., \& KinTsCh, W. (1985). Propositional and situational representations of text. Journal of Memory \& Language, 24, 503-518. Siegel, S., \& CASTELlaN, N. J. (1988). Nonparametric statistics for the behavioral sciences. New York: McGraw-Hill.

TAYLOR, H. A., \& TVERSKY, B. (1992). Spatial mental models derived from survey and route descriptions. Journal of Memory \& Language, 31, 261-292.

\section{NOTES}

1. All significance tests in this article were performed at the .05 level. 2. Because conclusions are being drawn on the basis of a null result, and because the pattern of cell means resembles the interactions found in Experiments 1 and $2 \mathrm{~A}-2 \mathrm{~B}$, one way of bolstering our conclusions is to perform an analysis across these experiments. That is, for performance on the preload version of the concurrent task, there should be a significant interaction contrast such that the average picture effect in Experiments 1 and 2A-2B (which included a concurrent dot task) exceeds the picture effect in Experiment 4 (in which subjects performed a concurrent digit task). Accordingly, the data from the preload concurrent task were subjected to the arcsine of the square root of $p$ transformation, which successfully induced homogeneity of variance, then analyzed by ANOVA. The interaction contrast was significant $[F(1,128)$ $\left.=5.95, M S_{e}=0.025\right]$ and accounted for $74 \%$ of the interaction sum of squares (SS).

3. This comparison can be examined explicitly in an analysis across experiments: the interaction contrast comparing the task effect in Experiment 4 with the average task effect observed in the other experiments was significant $\left[F(1,128)=8.23, M S_{\mathrm{e}}=146.89\right]$, and accounted for $84 \%$ of the SS for the task by experiment interaction.

\section{REFERENCES}

BADDELEY, A. (1986). Working memory. Oxford: Oxford University Press.
(Manuscript received June 8, 1993; revision accepted for publication September 3, 1993.) 